\title{
Joint detection scheme for cooperative spectrum sensing in cognitive radio network
}

\author{
Yijiang Nan ${ }^{*}$ (D), Chenglin Zhao and Bin Li
}

\begin{abstract}
In this paper, a new architecture of cognitive radio network (CRN) is presented for future dynamic spectrum sharing in time-variant flat fading (TVFF) channels. We consider a practical scenario where secondary users (SUs) are able to access the idle spectrum by secondary user routers (SU_Rs). Managed by a fusion center (FC), SU_Rs can work together to capture the idle spectrum, and then assign to the SUs. Besides, it is imperative to guarantee the wireless communication quality between primary base station (P_BS) and SU_Rs. Therefore, a new cooperative spectrum sensing (CSS) algorithm is suggested to recursively estimate the channel state information (CSI) while capturing the idle licensed band. The united mathematics model relies on a dynamic state-space model (DSM) and a Bernoulli filters (BF) algorithm. TVFF channels are modeled as finite-state Markov channel (FSMC). In order to reduce complexity of CSS, the particles are manipulated and reconstructed. Experimental simulations demonstrate that, by exploiting dynamic CSI, sensing performance of the new CSS algorithm will surpass the traditional schemes and this new architecture can be used in a realistic spectrum sharing system.
\end{abstract}

Keywords: Cognitive radio network, Cooperative spectrum sharing, Time-variant flat fading, Dynamic state-space model, Bernoulli filters

\section{Introduction}

In radar sensor networks, recent advances on waveform design and diversity $[1,2]$ have made spectrum sensing possible for applications such as radar sensor selections [3] and target detection [4]. Similarly, spectrum sensing is also possible for wireless networks. With tremendous growth in the wireless network market, the number of subscribers and the demand for high data rate have escalated greatly so as to strengthen the scarcity of spectrum resource in wireless networks [5]. However, the traditional approach of fixed spectrum allocation to a licensed network leads to the spectrum underutilization. According to the report from Federal Communications Commission (FCC), the spectrum utilization in the usage of allocated spectrum is as low as $15 \%$ [6]. As a result, this defect motivates the development of cognitive radio network (CRN) that allows secondary users to seek idle licensed bands cooperatively and share them

\footnotetext{
* Correspondence: nanyj@bupt.edu.cn

School of Information and Communication Engineering (SICE), Beijing

University of Posts and Telecommunications (BUPT), Beijing 100876, China
}

dynamically. Without causing serious interference to the primary users (PUs), such dynamic spectrum sharing will considerably improve the frequency utilization [7]. In fact, it has been widely adopted by both the FCC policy initiatives and IEEE standardizations, such as IEEE 802.22 [8] and IEEE 802.11k [9].

Despite enhancement of spectrum utilization, the transmission from secondary users (SUs) can interfere with the PU negatively. To guarantee interference-free spectrum access, the SUs are required to monitor the primary spectrum so as to capture the idle time in PU transmissions (also called spectrum holes). In CRN, cooperative spectrum sensing (CSS), participated by a group of spatially distributed SUs, has been widely adopted in previous sensing algorithms [10-12]. Compared with a single cognitive radio, CSS algorithms use multiple sensors and combine their measurements into one decision. Thus, CSS can overcome some limitations [7] of single spectrum sensing like energy constraints, and they are more suitable for the CRN, which consists of multiple nodes.

\section{苗 Springer}

(c) 2016 Nan et al. Open Access This article is distributed under the terms of the Creative Commons Attribution 4.0 International License (http://creativecommons.org/licenses/by/4.0/), which permits unrestricted use, distribution, and reproduction in any medium, provided you give appropriate credit to the original author(s) and the source, provide a link to the Creative Commons license, and indicate if changes were made. 
In Fig. 1, this paper designs a new architecture of CRN, where sensing modules of each $\mathrm{SU}$ can be separated and centralized by a fusion center (FC). In this case, spectrum sensing will be manipulated by FC, and SUs use the idle licensed band without reference to sensing process. To illustrate, this new architecture consists of a FC and a set of secondary user routers (SU_Rs), which play the role of sensing. In the cover region of a primary base station (P_BS), the SU_Rs cooperate with one another to detect the idle spectrum. In the cover region of each SU_R, SUs may communicate with the SU_R by a classical wireless technique, e.g., $802.11 \mathrm{n}$. As soon as there is enough idle bands, the SU_R will forward the data from SUs to the P_BS.

We consider the time-variant flat fading (TVFF) channels in the CRN. For the considered scenario, TVFF channels may deteriorate the sensing performance and reduce the quality of wireless communication between P_BS and SU_Rs. Therefore, in realistic TVFF scenarios, besides the idle licensed band, time-variant channel state information (CSI) on the cognitive links, as the important network information, needs to be estimated to ensure good sensing performance and helps SU_Rs acquire a better communication quality. Therefore, it is essential to propose a new CSS algorithm, which does not merely detect the PU and estimate the varying CSI jointly but also can be applicable to the new architecture.

However, most existing sensing algorithms are less attractive. With boosting the uncertainty on CSI, TVFF will remarkably degrade the sensing performance. Although some existing schemes take statistical property of fading effects into account [13], they can just reduce the detrimental effects to some extent but fail to track the evolution of such dynamic TVFF channels. From another aspect, since the received signals from P_BS will be disappeared intermittently, CSI is also hard to be acquired in CRN systems. Some feasible techniques such as machine learning methods [14] have been adopted; nevertheless, a separated CSI estimation will increase computational complex considerably, which limits their applications.

To confront with the above challenges, a new CSS algorithm is proposed. The main contributions are threefold.

1. We establish a dynamic state-space model (DSM) to characterize the process of change in CRN by Markov process, including the PU work state and the varying TVFF channels. With the scope of an ordinary base station, the synchronization can be obtained by Global Positioning System (GPS) [15], thus assuming that the synchronization and a priori pilot can be available. The matched filter (MF) method, as the optimal observed output, is used as the measurement.

2. Considering the measurement determined by both the PU work state and CSI, the time evolution Bernoulli random finite set (BRFS) is adopted to characterize the complex DSM procedure. Based on the Bayes theory and sequential estimation schemes, the cardinality (i.e., PU work state) and elements (i.e., CSI) of BRFS are estimated recursively. Particle filter is adopted to implement the above scheme.

3. As the number of SU_R increases, more dimensions of CSI vector will render the exponential growth of complexity. To combat this challenge, we further manipulate the construction of particles and rebuild new particles, based on the independence of channels. In this way, the computation burden of

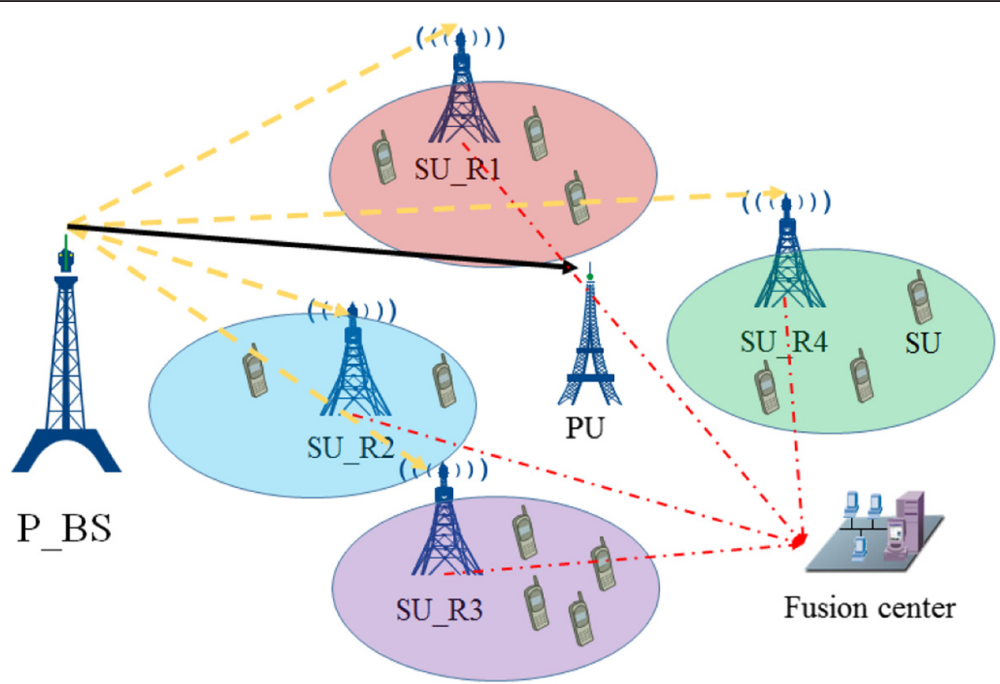

Fig. 1 Architecture of CRN. Yellow dotted line stands for cognitive links; black solid line strands for primary link 
an existing joint estimation-based sensing scheme can be greatly alleviated.

The remainder of this work is organized as follows. The DSM is designed in Section 2. Section 3 presents the CSS algorithm, a two-step BF mechanism, which has its foundation in the Bayesian theory and the BRFS. In Section 4, the implementation of each step and manipulation of particles will be described in detail. In Section 5, compared numerical simulations and performance analysis are provided. Finally, we conclude the investigation in Section 6.

\section{System model}

The stochastic DSM is adopted for describing the dynamic complex CRN system as follows:

$$
\begin{aligned}
& s_{n}=S\left(s_{n-1}\right) \\
& \boldsymbol{\alpha}_{n}=H\left(\boldsymbol{\alpha}_{n-1}\right) \\
& \mathbf{y}_{n}=G\left(\boldsymbol{\alpha}_{n}, s_{n}, \mathbf{d}_{n, m}, \mathbf{z}_{n, m}\right)
\end{aligned}
$$

The block diagram of DSM is depicted in Fig. 2. We define totally three equations for the DSM, including two dynamic equations $S($.$) and H($.) and one measurement equation $G$ (.). The transitional function $S$ (.) characterizes the stochastic evolution of the PU's state $s_{n} \in \mathbf{S}=\{0,1\}$ of the $n$th discrete time as the first-order Markov process. The other transition function $H($.$) spe-$ cifies dynamic behaviors of CSI vector $\boldsymbol{\alpha}_{n}=\left[\alpha_{0, n}, \ldots\right.$, $\left.\alpha_{u, n}, \ldots, \alpha_{U-1, n}\right]^{T}(u=0, \ldots, U-1)$, where $\alpha_{u, n}$ denotes the channels between P_BS and $u$ th SU_R. The observation function $G($.$) then describes \mathbf{y}_{n}=\left[y_{0, n}, \ldots, y_{u, n}, \ldots\right.$, $\left.y_{U-1, n}\right]^{T}(u=0, \ldots, U-1)$, where $y_{u, n}$ denotes the signal through matched filter of $u$ th SU_R and $\mathbf{d}_{n, m}=\left[d_{n, 0}, \ldots\right.$, $\left.d_{n, m}, \ldots, d_{n, M-1}\right]^{\mathrm{T}}$ denotes the pilot sequence signal of PU at $n$th discrete time, where $M$ signifies the length of pilot sequence. The random noise is involved in measurement process, and denoted by $\mathbf{z}_{n, m}=\left[z_{0, n, m}, \ldots\right.$, $\left.z_{u, n, m}, \ldots, z_{U-1, n, m}\right]^{\mathrm{T}}$, which is viewed as zero-mean additive white Gaussian noise (AWGN).
Additionally, three assumptions are made in the DSM for the ease of analysis.

1. The distance between different SU_Rs is far enough so that the elements of CSI vector $\alpha_{n}$ are of little correlation. Therefore, $\alpha_{0, n}, \ldots, \alpha_{u, n}, \ldots, \alpha_{U-1, n}$ are assumed to be independent and identically distributed (i.i.d.) variables.

2. In a slow-varying case, each fading channel gain $\alpha_{u, n}$ is assumed to remain unchanged with $L$ successive sensing slots. The static duration $L$ is related with the maximum Doppler frequency shift $f_{D}$, which is inversely proportional to $L$. Because of asynchrony of channel and the PU work state, we assume n' represents the changing slot of $\alpha_{u, n}$. For connection of two time index, $l$ is defined as the $l$ th $(0<l<L)$ PU switching slot in the slot $n^{\prime}$; thus, $n=L\left(n^{\prime}-1\right)+l$.

3. The PU's state is assumed to keep invariant in each sensing slot.

\subsection{Evolution of the PU work state}

The PU work state is of two forms: active and idle, and it is modeled as a two-value variable $s_{n} \in \mathbf{S}$, where $s_{n}=1$ denotes the active state and $s_{n}=0$ represents the other one, respectively. Survival probability $p_{\mathrm{s}}=\operatorname{Pr}\left\{s_{n+1}=\right.$ $\left.1 \mid s_{n}=1\right\}$ represents the PU work state that remains an active state from the slot $n$ to slot $n+1$. Similarly, birth probability $p_{\mathrm{b}}=\operatorname{Pr}\left\{s_{n+1}=1 \mid s_{n}=0\right\}$ represents switching active state. Therefore, the transitional probability matrix of the PU work state is given by

$$
\Pi=\left[\begin{array}{cc}
1-p_{\mathrm{b}} & p_{\mathrm{b}} \\
1-p_{\mathrm{s}} & p_{\mathrm{s}}
\end{array}\right]
$$

\subsection{Cognitive TVFF channel model}

In CRN, wireless propagation suffers from white Gaussian noises and TVFF. In this investigation, we fully make use of the finite-state Markov channel (FSMC) [16] to specify the cognitive channels because its Markov property can

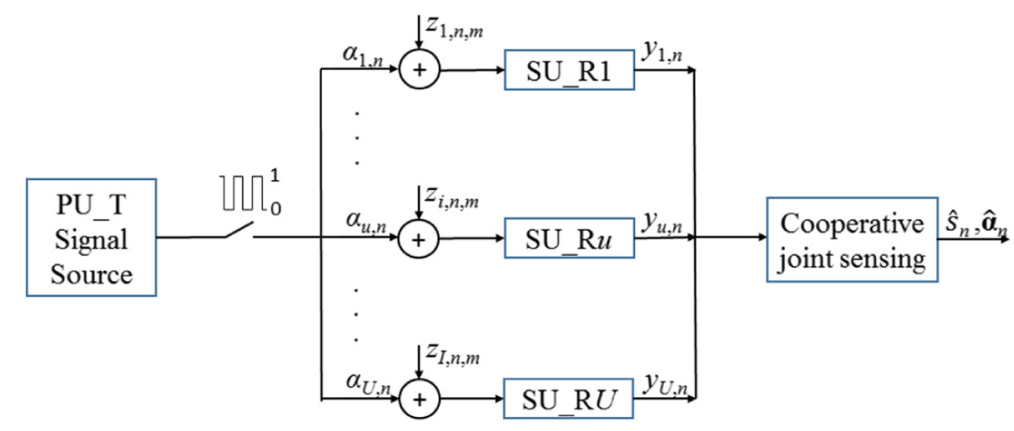

Fig. 2 Architecture of CRN; primary user transit $\left(P U_{-} T\right)$ 
effectively characterize the dynamic property of TVFF and match its statistical model.

In the FSMC model, the fading channel is divided into $K$ discrete states and each state transfers to others with specified probability. Let $A_{k} \in \mathbf{A}=\left\{A_{0}, A_{2}, \ldots, A_{K-1}\right\}$ denotes each channel state. Correlatively, the state transition can be characterized by a transitional probability matrix $\Pi_{K} \times{ }_{K}=\left\{\pi_{i \rightarrow j}, i, j \in 0,1, \ldots, K-1\right\}$ :

$$
\boldsymbol{\Pi}_{K \times K}=\left[\begin{array}{cccc}
\pi_{0 \rightarrow 0} & \pi_{0 \rightarrow 1} & \cdots & \pi_{0 \rightarrow(K 1)} \\
\pi_{1 \rightarrow 0} & \pi_{1 \rightarrow 1} & \cdots & \pi_{1 \rightarrow(K-1)} \\
\vdots & \vdots & \ddots & \vdots \\
\pi_{(K-1) \rightarrow 0} & \pi_{(K-1) \rightarrow 1} \cdots & \pi_{(K-1) \rightarrow(K-1)}
\end{array}\right]
$$

where $\pi_{k^{\prime} \rightarrow k}$ accounts for the transitional probability from the state $k^{\prime}$ at $n^{\prime}-1$ (channel slot index) to the state $k$ at $n$ :

$$
\pi_{k^{\prime} \rightarrow k}=\operatorname{Pr}\left\{a_{n^{\prime}}=A_{k} \mid a_{n^{\prime}-1}=A_{k^{\prime}}\right\}
$$

Considering the indecomposable FSMC model, evolution of channel is taken as a stationary Markov process. We denote stationary probability vector as $\pi=\left[\pi_{0}, \ldots\right.$, $\left.\pi_{k}, \ldots, \pi_{K-1}\right]^{T}$, where $\pi_{k}=\operatorname{Pr}\left\{\alpha_{n^{\prime}}=A_{k}\right\}$ is stationary distribution of channel state $A_{k}$, and this vector can be solved by $\Pi_{K} \times{ }_{K}^{T} \boldsymbol{\pi}=\boldsymbol{\pi}$. The nonnegative channel amplitude will be partitioned into $K$ non-overlapping regions: $V=\left\{\left[v_{0}, v_{1}\right]\right.$, $\left.\left[v_{1}, v_{2}\right], \ldots,\left[v_{K-1}, v_{K}\right]\right\}$. Suppose the PDF of fading channel (e.g., Rayleigh or Rican distribution) $f(\alpha)$, the steady probability of channel states can be computed by integrating between the region $\left[v_{k-1}, v_{k}\right]$ :

$$
\pi_{k}=\int_{v_{k-1}}^{v_{k}} \alpha f(\alpha) d \alpha
$$

Under the equal partition property $\pi_{k}=1 / K$, the partitioning bounds are easily derived from

$$
v_{k}=\sqrt{-2 \sigma^{2} \cdot \ln \left(1-^{k} / K\right)}
$$

Simplistically, we adopt a first-order FSMC which is fully enough to match with statistical fading models (e.g., Clarke's model [16]). Then, the transitional probability from channel state $A_{k}$ to $A_{k}$ can be determined by

$$
\begin{aligned}
\pi_{k^{\prime} \rightarrow k} & =\frac{1}{\pi_{k}} \times \int_{v_{k^{\prime}}}^{v_{k^{\prime}+1}} \int_{v_{k}}^{v_{k+1}} f\left(\alpha_{n^{\prime}-1}, a_{n^{\prime}}\right) d \alpha_{n^{\prime}-1} d a_{n^{\prime}}, \\
k & =0,1, \ldots K-1
\end{aligned}
$$

where $f\left(\alpha_{n^{\prime}-1}, \alpha_{n^{\prime}}\right)$ is the bivariate joint PDF.

\subsection{Observation model}

For implementation with ease, we adopt a matched filter sensing scheme [17] for reference. Each SU_R observing system is independent with the others. Therefore, the spectrum sensing can be formulated to the following hypothesis test problem in each SU_R:

$$
\begin{aligned}
y_{u, n} & =\sum_{m=1}^{M}\left(\alpha_{u, n} s_{n} d_{n, m}+z_{u, n, m}\right) d_{n, m} \\
& = \begin{cases}\sum_{m=1}^{M} z_{u, n, m} d_{n, m} & H_{0} \\
\sum_{m=1}^{M}\left(\alpha_{u, n} d_{n, m}+z_{u, n, m}\right) d_{n, m} & H_{1}\end{cases}
\end{aligned}
$$

Here, $H_{0}$ and $H_{1}$ represent two opposite hypotheses, respectively, i.e., idle work state and active work state; $M$ is the length of pilot signal; $y_{u, n} \in \mathbf{y}_{n}$ is the measurement in the $u$ th SU_R; $d_{n, m} \in \mathbf{d}_{n, m}$ is the amplitude of pilot signal in $n$th sensing slot; and the AWGN is $z_{u, n, m} \in \mathbf{z}_{n, m}$ with zero mean and a variance of $\sigma_{n}^{2}$, i.e., $z_{u, n, m} \sim N\left(0, \sigma_{n}^{2}\right)$.

Due to the received signals at different SU_Rs are independent with one another, the likelihood function $\varphi\left(\mathbf{y}_{n} \mid s_{n}, \mathbf{\alpha}_{n}\right)$ may follow joint distribution, consisting of $\varphi_{u}\left(y_{u, n} \mid s_{n}, \alpha_{u, n}\right)(u=0, \ldots, U-1)$ :

$$
\varphi\left(\mathbf{y}_{n} \mid s_{n}, \mathbf{\alpha}_{n}\right)=\prod_{u=0}^{U-1} \varphi_{u}\left(y_{u, n} \mid s_{n}, \alpha_{u, n}\right)
$$

Conditioned on the different fading channel $\alpha_{u, n}$ and the PU work state $s_{n}$, sub-likelihood function $\varphi_{u}\left(y_{u, n} \mid s_{n}, \alpha_{u, n}\right)$ may follow a Gaussian distribution with zero mean under $H_{0}\left(s_{n}=0\right)$, and with none-zero mean under $H_{1}\left(s_{n}=1\right)$ :

$$
\varphi_{u}\left(y_{u, n} \mid s_{n}, \alpha_{u, n}\right)= \begin{cases}\frac{1}{\sqrt{2 \pi M \sigma^{2}}} \exp \left(\frac{y_{u, n}^{2}}{2 M \sigma^{2}}\right), & H_{0} \\ \frac{1}{\sqrt{2 \pi M \sigma^{2}}} \exp \left(\frac{\left(y_{u, n}-M \alpha_{u, n}\right)^{2}}{2 M \sigma^{2}}\right), & H_{1}\end{cases}
$$

where $\sigma^{2}$ represents the variance of Gaussian noise.

\section{Joint cooperative spectrum sensing algorithm}

In classical CSS schemes, i.e., optimal soft combination scheme [10], such combination of detection and estimation will not be implemented, since most of them are also threshold-based techniques, which only solve detection issues. Therefore, a Bayesian stochastic approach is an effective solution to estimate such two parameters jointly. Our task is to estimate recursively the posterior PDF of the state $\mathbf{s}_{n}$, which is viewed as $\mathbf{s}_{n}=\left\{s_{n}, \boldsymbol{\alpha}_{n}\right\}$.

\subsection{Optimal soft combination scheme}

An optimal soft combination scheme [10] estimates the PU work state by threshold decision. Without loss of generality, the classical scheme is concerned with 
maximizing the detection probability for a given false alarm probability. Thus, the Neyman-Pearson (NP) criterion is applied, which is equivalent to the likelihood ratio test. The corresponding likelihood ratio $L R\left(\mathbf{y}_{n}\right)$ between two hypotheses and decision function are expressed as

$$
L R\left(\mathbf{y}_{n}\right)=\frac{\operatorname{Pr}\left(\mathbf{y}_{n} \mid H_{1}\right)}{\operatorname{Pr}\left(\mathbf{y}_{n} \mid H_{0}\right)}=\prod_{u=0}^{U-1} \frac{\operatorname{Pr}\left(y_{u, n} \mid H_{1}\right)}{\operatorname{Pr}\left(y_{u, n} \mid H_{0}\right)} \gtrless_{H_{0}}^{H_{1}} h
$$

where $h$ is the threshold determined by the given false alarm.

In this considered scenario, SU_Rs send their original sensing information to the fusion center without any local processing, and then the measurements are accumulated with its weights appropriately. According to eqs. (11)-(12), based on the NP criterion, threshold judgment is accomplished by the likelihood ratio $L R\left(\mathbf{y}_{n}\right)$ which is expressed as

$$
L R\left(\mathbf{y}_{n}\right)=\prod_{u=0}^{u-1} \frac{\operatorname{Pr}\left(y_{u, n} \mid H_{1}\right)}{\operatorname{Pr}\left(y_{u, n} \mid H_{0}\right)}=\exp \left(\sum_{u=0}^{u-1} \frac{\alpha_{u, n} y_{u, n}}{\sigma^{2}}-\sum_{u=0}^{U-1} \frac{M \alpha_{u, n}^{2}}{2 \sigma^{2}}\right)
$$

Therefore, the original decision criterion given in eq. (13) is rewritten as

$$
y_{n}^{\prime}=\sum_{u=0}^{U-1} w_{u, n}^{\prime} y_{u, n}=\sum_{u=0}^{U-1} \frac{\sqrt{\gamma_{u, n}}}{\sigma} y_{u, n} \underset{H_{0}}{\stackrel{H_{1}}{\gtrless}} \ln (h)+\sum_{u=1}^{I} \frac{1}{2} \gamma_{u, n} M
$$

where $y_{n}^{\prime}$ is the total accumulated measurement as the weight summation of different SU_Rs, whose weight $w_{u, n}^{\prime}$ is corresponding to its instantaneous signal to noise ratio (SNR) $\gamma_{u, n}$ at time slot $n$. Thus, we obtain the optimal soft combination in our DSM.

\subsection{Bayesian scheme}

From the Bayes theory view, given a prior transitional function $p\left(\mathbf{s}_{n} \mid \mathbf{s}_{n-1}\right)$ and the likelihood function $\varphi$ $\left(\mathbf{y}_{n} \mid \mathbf{s}_{n}\right)$, the joint posterior distribution is then propagated sequentially via the well-known two-step procedure, assuming that initial density of the state, $p\left(\mathbf{s}_{0}\right)$ is known.

1. The first-predicting step will be integrated via the Chapman-Kolmogorov equation as follows:

$p_{n \mid n-1}\left(\mathrm{~s}_{n} \mid \mathrm{y}_{0: n-1}\right)=\int p_{n-1 \mid n-1}\left(\mathrm{~s}_{n-1} \mid \mathrm{y}_{0: n-1}\right) \cdot \phi\left(\mathrm{s}_{n} \mid \mathrm{s}_{n-1}\right) d \mathrm{~s}_{n-1}$

where $p_{n-1 \mid n-1}\left(\mathrm{~s}_{n-1} \mid \mathrm{y}_{0: n-1}\right)$ denotes the posterior density of $\mathrm{s}$ at $n-1$ th slot and transitional function $\phi\left(\mathbf{s}_{n} \mid \mathbf{s}_{n-1}\right)$ can be decomposed as

$$
\begin{aligned}
\phi\left(\mathbf{s}_{n} \mid \mathbf{s}_{n-1}\right)= & \operatorname{Pr}\left(s_{n} \mid s_{n-1}\right) \\
& \times \prod_{u=0}^{U-1} \pi_{n \mid n-1}\left(\alpha_{u, n} \mid \alpha_{u, n-1}\right), \quad u=0,1, \ldots, U-1
\end{aligned}
$$

2 The second-updating step applies the Bayes rule to update the predicted PDF. Taking measurement vector $\mathrm{y}_{n}$ into account, current posterior density can be expressed as

$p_{n \mid n}\left(\mathrm{~s}_{n} \mid \mathrm{y}_{0: n}\right)=\frac{p_{n \mid n-1}\left(\mathrm{~s}_{n} \mid \mathrm{y}_{0: n-1}\right) \cdot \varphi\left(\mathrm{y}_{n} \mid \mathrm{s}_{n}\right)}{\int p_{n \mid n-1}\left(\mathrm{~s}_{n} \mid \mathrm{y}_{0: n-1}\right) \cdot \varphi\left(\mathrm{y}_{n} \mid \mathrm{s}_{n}\right) d \mathrm{~s}_{n}}$

Knowing the posterior $p_{n \mid n}\left(\mathrm{~s}_{n} \mid \mathrm{y}_{0: n}\right)$, we can estimate the CSI and PU work state according to maximum A posteriori (MAP) criterion.

\subsection{Bernoulli random finite sets}

Despite the independence of two estimated variables, we cannot just model them simply as $\mathbf{s}_{n}=\left[s_{n}, \mathbf{\alpha}_{n}\right]^{T}$ since the measurement $\mathbf{y}_{n}$ is not made of multiplication. The likelihood function of $\mathbf{y}_{n}$ switches alternately along with appearance/disappearance of PU. Thus, BRFS [18] $\mathscr{F}$, as a stochastic variable that takes a value as an empty or singleton set, is fully suitable for the construction of state $\mathbf{s}_{n}$.

The cardinality of $\mathscr{F}$ is random and modeled by a Bernoulli distribution $\rho(k)=\operatorname{Pr}\{|\mathscr{P}|=k\}, k \in\{0,1\}$, and it can be defined as a PU work state. Meanwhile, its element is completely specified by a joint distribution $p_{k}\left(\left\{\boldsymbol{\alpha}_{0}\right.\right.$ $\left.\ldots \boldsymbol{\alpha}_{k} \ldots \boldsymbol{\alpha}_{K-1\}}\right\}$ conditioned on cardinality $k$ and can stand for CSI vector $\boldsymbol{\alpha}_{n}$, whose distribution is related to the TVFF. In order to describe the statistical nature of such $\mathscr{F}$, we adopt Mahler's approach [18], finite set statistics (FISST) PDF, to specify BRFS. This $n$th PDF is uniquely determined by $\rho(k)$ and $p_{n}\left(\boldsymbol{\alpha}_{n}\right)$ :

$$
p_{F}\left(\mathcal{F}_{n}\right)= \begin{cases}q \cdot p_{n}\left(\alpha_{n}\right) & \mathcal{F}_{n}=\left\{\alpha_{n}\right\} \\ 1-q & \mathcal{F}_{n}=\varnothing\end{cases}
$$

where $\mathscr{F}$ is empty with probability $1-q$ or has a singleton element with probability $q$. The set integral is defined as

$$
\int_{\mathcal{F}} p_{\mathcal{F}}\left(\mathcal{F}_{n}\right) \delta \mathcal{F}=p_{\mathcal{F}}(\varnothing)+\int p_{\mathcal{F}}\left(\alpha_{n}\right) d \alpha_{n}
$$

Here, eq. (20) denotes the set integration on $\mathscr{F}$ and it integrates to one. Correspondingly, transitional function of $\mathscr{F}$ has to suit to its architecture. Due to four state transitions in eq. (4), given the different PU work states at the slot $n-1$, we can write the priori transitional density: 


$$
\begin{gathered}
\phi_{n \mid n-1}\left(\mathcal{F}_{n} \mid \varnothing\right)= \begin{cases}1-p_{b} & \left|\mathcal{F}_{n}\right|=0 \\
p_{b} \cdot b_{n \mid n-1}\left(\alpha_{n}\right) & \left|\mathcal{F}_{n}\right|=1 \\
0 & \left|\mathcal{F}_{n}\right| \geq 2\end{cases} \\
\phi_{n \mid n-1}\left(\mathcal{F}_{n} \mid\left\{\alpha_{n-1}\right\}\right)=\left\{\begin{array}{cc}
1-p_{s} & \left|\mathcal{F}_{n}\right|=0 \\
p_{s} \cdot \pi_{n \mid n-1}\left(\alpha_{n} \mid \alpha_{n-1}\right) & \left|\mathcal{F}_{n}\right|=1 \\
0 & \left|\mathcal{F}_{n}\right| \geq 2
\end{array}\right.
\end{gathered}
$$

where $\pi_{n \mid n-1}($.$) is the transitional density of CSI \boldsymbol{\alpha}_{n}$ obtained from the FSMC model and $b_{n \mid n-1}($.$) is the birth$ density, which represents the initial density of CSI $\boldsymbol{\alpha}_{n}$ when the PU is reactive.

\subsection{Bernoulli filters algorithm}

A stochastic algorithm, named Bernoulli filters, is a useful tool to estimate the posterior FISST PDF of BRFS recursively. According to eq. (19) above, the FISST PDF of BRFS $\mathscr{F}_{n}$ at $n$th slot is made up of two important distributions. One is the posterior density of PU's appearance $q_{n \mid n}$. The other one is the posterior spatial PDF of the CSI $\boldsymbol{\alpha}_{n,}, f_{n \mid n}\left(\boldsymbol{\alpha}_{n}\right)$.

With the well-known two-step procedure of Bayesian estimation framework, Bernoulli filters (BF) [19], as a sequential Bayesian estimator, propagates two posterior terms recursively. The specific approach will also be divided into predict step and update step.

\section{Predict step}

It is assumed that the posterior FISST PDF of BRFS $\mathscr{F}_{n-1}$ is $\left(p_{n-1 \mid n-1} \mathscr{F}_{n-1} \mid \mathrm{y}_{n-1}\right)$ at $n-1$ th slot, which includes two terms $q_{n-1 \mid n-1}$ and $f_{n-1 \mid n-1}\left(\alpha_{n}\right)$ above. Then, the predicted FISST PDF of BRFS $\left(p_{n \mid n-1} \widetilde{\mathscr{F}}_{n-1} \mid \mathrm{y}_{n-1}\right)$ has been derived originally from eq. (16). Based on the Mahler approach eq. (20), $\left(p_{n \mid n-1} \widetilde{\mathscr{T}}_{n} \mid \mathrm{y}_{n-1}\right)$ is derived from

$$
\begin{aligned}
& p_{n \mid n-1}\left(\mathcal{F}_{n} \mid \mathrm{y}_{n-1}\right) \\
& =\int \phi_{n \mid n-1}\left(\mathcal{F}_{n} \mid \mathcal{F}_{n-1}\right) p_{n-1 \mid n-1}\left(\mathcal{F}_{n-1} \mid \mathrm{y}_{n-1}\right) \delta \mathcal{F}_{n-1} \\
& =\phi_{n \mid n-1}\left(\mathcal{F}_{n} \mid \varnothing\right) p_{n-1 \mid n-1}\left(\varnothing \mid \mathrm{y}_{n-1}\right) \\
& +\int \phi_{n \mid n-1}\left(\mathcal{F}_{n} \mid\left\{\alpha_{n-1}\right\}\right) p_{n-1 \mid n-1}\left(\left\{\alpha_{n-1}\right\} \mid \mathrm{y}_{n-1}\right) d \alpha_{n-1}
\end{aligned}
$$

By viewing its cardinality, we consider two different cases, i.e., $\widetilde{\mathscr{F}}_{n}=\varnothing$ and $\widetilde{\mathscr{F}}_{n}=\left\{\boldsymbol{\alpha}_{n}\right\}$. Two predictions $q_{n \mid n-1}$ and $f_{n \mid n-1}\left(\boldsymbol{\alpha}_{n}\right)$ of $p_{n \mid n-1}\left(\boldsymbol{F}_{n} \mid \mathbf{y}_{n-1}\right)$, based on the FISST PDF of BRFS in eq. (19), can be simplified as

$$
\begin{aligned}
& q_{n \mid n-1}=p_{b}\left(1-q_{n-1 \mid n-1}\right)+p_{s} q_{n-1 \mid n-1} \\
& f_{n \mid n-1}\left(\boldsymbol{\alpha}_{n}\right)=\frac{p_{b}\left(1-q_{n-1 \mid n-1}\right) b_{n \mid n-1}\left(\boldsymbol{\alpha}_{n}\right)}{q_{n \mid n-1}} \\
& +\frac{p_{s} q_{n-1 \mid n-1} \int \pi_{n \mid n-1}\left(\boldsymbol{\alpha}_{n} \mid \mathbf{\alpha}_{n-1}\right) \cdot f_{n-1 \mid n-1}\left(\boldsymbol{\alpha}_{n-1}\right) d \mathbf{\alpha}_{n-1}}{\left.\mathrm{q}_{n}\right|_{n-1}}
\end{aligned}
$$

where $f_{n \mid n-1}\left(\boldsymbol{\alpha}_{n}\right)$ consists of two parts, i.e., birth part and survival part.

\section{Update step}

The updated processing of BF for the MF-based measurement vector $\mathrm{y}_{n}$ has been derived from eq. (10). Recalling from predicted FISST PDF of BRFS $\left(p_{n \mid n-1} \mathscr{F}_{n} \mid \mathrm{y}_{n-1}\right)$, the updated FISST PDF of BRFS $\left(p_{n \mid n} \mathscr{F}_{n} \mid \mathrm{y}_{n}\right)$ is based on the Bayes rule:

$$
p_{n \mid n}\left(\mathscr{F}_{n} \mid \mathrm{y}_{0: n}\right)=\frac{p_{n \mid n-1}\left(\mathscr{F}_{n} \mid \mathrm{y}_{0: n-1}\right) \cdot \varphi\left(\mathrm{y}_{n} \mid \mathscr{F}_{n}\right)}{p\left(\mathrm{y}_{n} \mid \mathrm{y}_{0: n-1}\right)}
$$

Using the standard Chapman-Kolmogorov function and set integration, the denominator of eq. (25) is simplified as

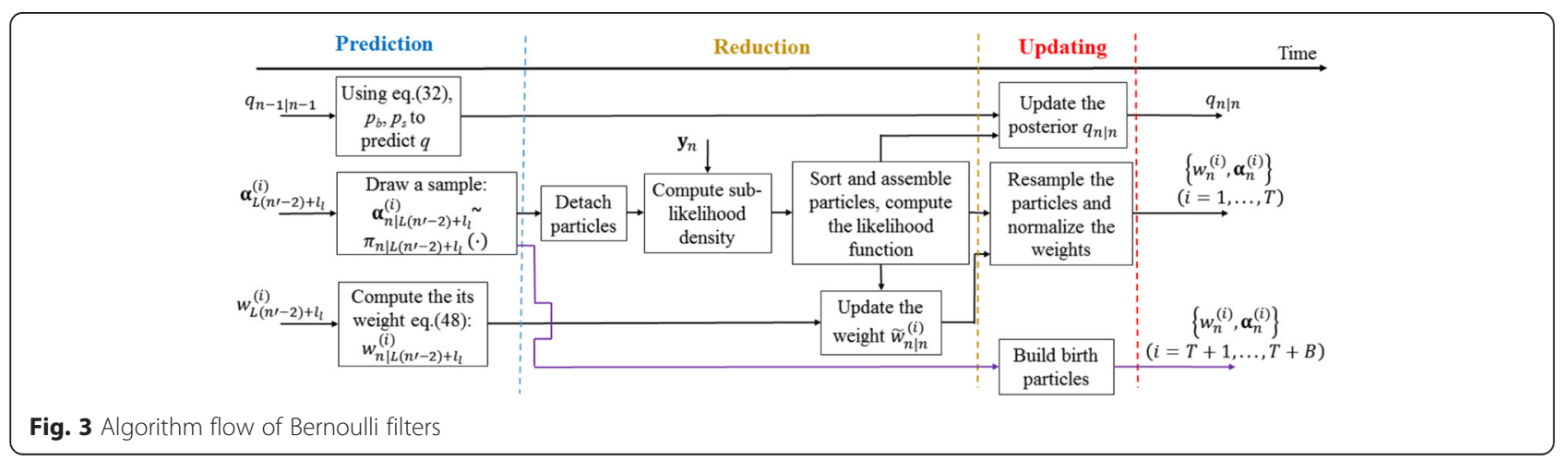




$$
\begin{aligned}
& p\left(\mathbf{y}_{n} \mid \mathbf{y}_{0: n-1}\right)=\int \phi\left(\mathbf{y}_{n} \mid \mathcal{F}_{n}\right) \cdot p_{n \mid n-1}\left(\mathcal{F}_{n} \mid \mathbf{y}_{1: n-1}\right) \delta \mathcal{F}_{n} \\
& =\left(1-q_{n \mid n-1}\right) \phi\left(\mathbf{y}_{n} \mid \varnothing\right)+q_{n \mid n-1} \int \phi\left(\mathbf{y}_{n} \mid\left\{\alpha_{n}\right\}\right) \cdot f_{n \mid n-1}\left(\alpha_{n}\right) d \alpha_{n}
\end{aligned}
$$

Similarly, the update step will also consider two cases of $\mathscr{F}_{n}$. Therefore, the current probability of PU appearance $q_{n \mid n}$ and spatial PDF $f_{n \mid n}\left(\boldsymbol{\alpha}_{n}\right)$ can be constructed as

$$
\begin{gathered}
q_{n \mid n}=\frac{q_{n \mid n-1} \int r_{n}\left(\mathbf{y}_{n} \mid\left\{\boldsymbol{\alpha}_{n}\right\}\right) f_{n \mid n-1}\left(\boldsymbol{\alpha}_{n}\right) d \mathbf{\alpha}_{n}}{1-q_{n \mid n-1}+q_{n \mid n-1} \int r_{n}\left(\mathbf{y}_{n} \mid\left\{\boldsymbol{\alpha}_{n}\right\}\right) f_{n \mid n-1}\left(\mathbf{\alpha}_{n}\right) d \mathbf{\alpha}_{n}} \\
f_{n \mid n}\left(\boldsymbol{\alpha}_{n}\right)=\frac{\varphi\left(\mathbf{y}_{n} \mid\left\{\mathbf{\alpha}_{n}\right\}\right) f_{n \mid n-1}\left(\boldsymbol{\alpha}_{n}\right)}{\int \varphi\left(\mathbf{y}_{n} \mid\left\{\boldsymbol{\alpha}_{n}\right\}\right) f_{n \mid n-1\left(\mathbf{\alpha}_{n}\right) d \mathbf{\alpha}_{n}}}
\end{gathered}
$$

where $r_{n}($.$) represents the measurement likelihood ratio$ between two hypotheses:

$$
r_{n}\left(\mathbf{y}_{n} \mid\left\{\alpha_{n}\right\}\right)=\frac{\varphi\left(\mathbf{y}_{n} \mid\left\{\alpha_{n}\right\}\right)}{\varphi\left(\mathbf{y}_{n} \mid \varnothing\right)}=\prod_{u=0}^{U-1} \frac{\varphi_{u}\left(y_{u, n} \mid s_{n}=1, \alpha_{u, n}\right)}{\phi_{u}\left(y_{u, n} \mid s_{n}=0, \alpha_{u, n}\right)}
$$

Note that eq. (28) is effectively the same as the conventional Bayesian estimation update eq. (18).

\section{Implementation}

Before implementation of $\mathrm{BF}$, some crucial problem should be taken fully into account. One problem is the asynchrony between the PU work state and CSI, which motivate us to refine the $\mathrm{BF}$ algorithm. On the other hand, how to eliminate the exponential growth of complexity associated with increasing number of SU_Rs is a key point for the application.

\subsection{Asynchrony of BF algorithm}

Since the channel state remains unchanged periodically, the channel state $\alpha_{u, n}$ will be evolved from the channel state over the last channel slot $n^{\prime}-1$, not from the channel state at slot $n-1$. Thus, the predicted function in eq. (24) will be reconstructed as where $l_{l}$ denotes the estimation of CSI $\boldsymbol{\alpha}_{n}$ in the final PU active state over the slot $n^{\prime}-1$. This handy manipulation solves non-synchronous problems successfully without any error because of the independence of such two terms, $q$ and $f_{n \mid n}\left(\boldsymbol{\alpha}_{n}\right)$.

\subsection{Implementation of Bernoulli filters}

PF provides a general framework for the implementation of BF (Fig. 3). This new framework approximates the posterior distribution of CSI $\boldsymbol{\alpha}_{n}, f_{n \mid n}\left(\boldsymbol{\alpha}_{n}\right)$, via a group of discrete particles $\left\{w_{n}^{(i)}, \boldsymbol{\alpha}_{n}^{(i)}\right\}(i=1, \ldots, T)$, where $\boldsymbol{\alpha}_{n}^{(i)}$ is the state of the particle of $f_{n \mid n}\left(\boldsymbol{\alpha}_{n}\right), T$ is the total number of particles, and $w_{n}^{(i)}$ are its weight. Since $f_{n \mid n}\left(\boldsymbol{\alpha}_{n}\right)$ is a probability density function, the weight is normalized and the sum of $w_{n}^{(i)}$ is equal to 1 .

Supposed at slot $n-1$, the probability of existence $q_{n-}$ $1 \mid n-1$ and the spatial PDF $f_{n-1 \mid n-1}\left(\boldsymbol{\alpha}_{n-1}\right)$ is approximated by

$$
f_{n-1 \mid} n-1\left(\boldsymbol{\alpha}_{n-1}\right)=\sum_{i=1}^{T} w_{n-1}^{(i)} \delta_{\boldsymbol{\alpha}_{n-1}^{(i)}}\left(\boldsymbol{\alpha}_{n-1}\right)
$$

where $\delta_{b}($.$) is the Dirac delta function that is concentrated$ at point $b$.

\section{Predicted step}

In the predicted step, the FISST PDF of $F_{n}$ is implemented as follows: the prior probability of the PU work state $q_{n \mid n-1}$ is easily straightforward; see eq. (23). According to eq. (30), the predicted step for $f_{n \mid L\left(n^{\prime}-2\right)+l l}\left(\alpha_{n}\right)$ involves the sum of two terms: birth and survival parts, which should be approximated numerically due to the intractable integration on the continuous distribution. Therefore, it can be described as

$$
f_{n \mid L\left(n^{\prime}-2\right)+l_{l}}\left(\alpha_{n}\right) \approx \sum_{i=1}^{T+B} w_{n \mid L\left(n^{\prime}-2\right)+l_{l}}^{(i)} \delta_{\alpha_{n \mid L\left(n^{\prime}-2\right)+l_{l}}^{(i)}}\left(\alpha_{n}\right)
$$

where $B$ denotes the number of birth particles. Two groups of particles above will be simulated accordingly from

$$
\begin{aligned}
f_{n \mid L\left(n^{\prime}-2\right)+l_{l}}\left(\mathbf{\alpha}_{L\left(n^{\prime}-2\right)+l_{l}}\right)= & \frac{p_{b}\left(1-q_{n-1 \mid n-1}\right) b_{n \mid L\left(n^{\prime}-2\right)+l_{l}}\left(\mathbf{\alpha}_{n}\right)}{q_{n \mid n-1}} \\
& +\frac{p_{s} q_{n-1 \mid n-1} \int \pi_{n \mid L\left(n^{\prime}-2\right)+l_{l}}\left(\mathbf{\alpha}_{n} \mid \mathbf{\alpha}_{L\left(n^{\prime}-2\right)+l_{l}}\right) \cdot f_{L\left(n^{\prime}-2\right)+l_{l} \mid L\left(n^{\prime}-2\right)+l_{l}}\left(\mathbf{\alpha}_{L\left(n^{\prime}-2\right)+l_{l}}\right) d \mathbf{\alpha}_{L\left(n^{\prime}-2\right)+l_{l}}}{q_{n \mid n-1}}
\end{aligned}
$$




$$
\begin{aligned}
& \alpha_{n \mid L\left(n^{\prime}-2\right)+l_{l}}^{(i)} \\
& =\left\{\begin{array}{lr}
\pi_{n \mid L\left(n^{\prime}-2\right)+l_{l}}\left(\alpha_{n \mid L\left(n^{\prime}-2\right)+l_{l}} \mid \alpha_{L\left(n^{\prime}-2\right)+l_{l}}^{(i)}, \mathrm{y}_{1: n-1}\right), & i=1, \ldots, T \\
\beta_{n}\left(\alpha_{n \mid L\left(n^{\prime}-2\right)+l_{l}} \mid \mathrm{y}_{1: n-1}\right), & i=T+1, \ldots, T+B
\end{array}\right.
\end{aligned}
$$

where birth particles are drawn from birth density $\beta_{n}($.$) . At the same time, we adopt prior transitional$ density of CSI $\alpha_{n}$ as the sequential importance sampling; thus, weight $w_{n-1}^{(i)}$, corresponding to particle $\alpha_{n}^{(i)}$, will be predicted according to [19]:

$$
w_{n \mid L\left(n^{\prime}-2\right)+l_{l}}^{(i)}= \begin{cases}\frac{p_{s} q_{n-1 \mid n-1}}{q_{n \mid n-1}} w_{L\left(n^{\prime}-2\right)+l_{l}}^{(i)}, & i=1, \ldots, T \\ \frac{p_{b}\left(1-q_{n-1 \mid n-1}\right)}{q_{n \mid n-1}} w_{L\left(n^{\prime}-2\right)+l_{l}}^{(i)}, & i=T+1, \ldots, T+B\end{cases}
$$

The transition of survival particles has been introduced in details according to transitional function. When it comes to the prior birth density of CSI $\boldsymbol{\alpha}_{n}$, $b_{n \mid L\left(n^{\prime}-2\right)+l l}\left(\boldsymbol{\alpha}_{n}\right)$, in absence of prior knowledge, a more effective way is to use the previous measurements to build adaptively birth particles [20], which follow the standard Chapman-Kolmogorov prediction the same as the survival transition in eq. (16):

$$
\begin{aligned}
& b_{n \mid L\left(n^{\prime}-2\right)+l_{l}}\left(\boldsymbol{\alpha}_{n} \mid \boldsymbol{\alpha}_{L\left(n^{\prime}-2\right)+l_{l}}\right) \\
& =\int \pi_{n \mid L\left(n^{\prime}-2\right)+l_{l}}\left(\boldsymbol{\alpha}_{n} \mid \boldsymbol{\alpha}_{L\left(n^{\prime}-2\right)+l_{l}}\right) \cdot b_{L\left(n^{\prime}-2\right)+l_{l}}\left(\boldsymbol{\alpha}_{L\left(n^{\prime}-2\right)+l_{l}}\right) d \boldsymbol{\alpha}_{L\left(n^{\prime}-2\right)+l_{l}}
\end{aligned}
$$

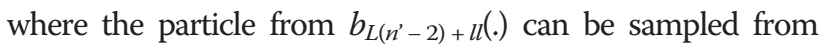
the prior transitional density $f_{L\left(n^{\prime}-2\right)+l l \mid L\left(n^{\prime}-3\right)+l l}\left(\mathbf{\alpha}_{L\left(n^{\prime}-2\right)+l l}\right)$ at slot $L\left(n^{\prime}-2\right)+l_{l}$ with equal weight $w_{L\left(n^{\prime}-2\right)+l l}^{(i)}=1 / B$.

2. Updated step

In the updated step, the processing of implementation is similar to the predicted one. The weight $w_{n \mid}^{(i)} L\left(n^{\prime}-2\right)+l l$ in eq. (34) is renewed by likelihood function:

$\tilde{w}_{n}^{(i)}=\varphi_{n}\left(\mathrm{y}_{n} \mid\left\{\alpha_{n \mid L\left(n^{\prime}-2\right)+l_{l}}^{(i)}\right\}\right) w_{n \mid L\left(n^{\prime}-2\right)+l_{l}}^{(i)}$

Then, the normalized weight of $i$ th particle is derived as

$w_{n}^{(i)}=\frac{\tilde{w}_{n}^{(i)}}{\sum_{i=1}^{T+B} \tilde{w}_{n}^{(i)}}$

Finally, $\alpha_{n}^{(i)}$ is equal to $\alpha_{L\left(n^{\prime}-2\right)+l l}^{(i)}$ with its corresponding weight $w_{n}^{(i)}$.
On the other hand, posterior density of PU's appearance $q_{n \mid n}$ is constructed recursively in eq. (27). In this equation, the integral of likelihood ratio and predicted function are approximated by

$$
\begin{aligned}
& I_{n}=\int r_{n}\left(\mathrm{y}_{n} \mid\left\{\alpha_{n}\right\}\right) f_{n \mid L\left(n^{\prime}-2\right)+l_{l}}\left(\alpha_{n}\right) d \alpha_{n} \\
& \approx \sum_{i=1}^{T+B} r_{n}\left(\mathrm{y}_{n} \mid\left\{\alpha_{n \mid L\left(n^{\prime}-2\right)+l_{l}}^{(i)}\right\}\right) w_{n \mid L\left(n^{\prime}-2\right)+l_{l}}^{(i)}
\end{aligned}
$$

Then, $q_{n \mid n}$ is obtained with ease.

\section{MAP estimation}

Based on the MAP, the threshold $\gamma_{n}$ at slot $n$ will be set up to 0.5 empirically and CSI $\alpha_{n}$ can be estimated by spatial PDF $f_{n \mid n}\left(\alpha_{n}\right)$ :

$$
\begin{aligned}
& \hat{s}_{n}= \begin{cases}1, & q_{n \mid n} \geq \gamma_{n}(\text { active }) \\
0, & q_{n \mid n}<\gamma_{n},(\text { idle })\end{cases} \\
& \hat{\alpha}_{n}=\arg \max _{\alpha_{i, n} \in \mathrm{A}} f_{n \mid n}\left(\alpha_{n}\right)
\end{aligned}
$$

\subsection{Reducing complexity}

In the CSS algorithm, the particle number $(T+B)$ conditions the accuracy of spatial PDF $f_{n \mid n}\left(\boldsymbol{\alpha}_{n}\right)$. In order to ensure the existence of each kind of particle's transition, all kinds of particles should be fully contained. With the increasing SU_R's number I, exponential growth of particles will deteriorate computational efficiency seriously.

According to a MAP criterion, we just need to find the optimal particle to maximize the spatial PDF $f_{n \mid n}\left(\mathbf{\alpha}_{n}\right)$ rather than approximate the whole PDF. Thus, in Fig. 4, we can manipulate particles via detaching, sorting, and assembling, which polarizes particles between best and worst. Based on independence of different channels, we can detach each particle of $f_{n \mid L\left(n^{\prime}-2\right)+l l}\left(\boldsymbol{\alpha}_{n}\right)$ in eq. (40),

$$
\begin{aligned}
f_{n \mid L\left(n^{\prime}-2\right)+l_{l}}\left(\boldsymbol{\alpha}_{n \mid L\left(n^{\prime}-2\right)+l_{l}}\right) \\
=\prod_{u=1}^{U} f_{u, n \mid L\left(n^{\prime}-2\right)+l_{l}}\left(\alpha_{u, n \mid L\left(n^{\prime}-2\right)+l_{l}}\right) \\
=\prod_{u=1}^{U} \sum_{i=1}^{T+B} w_{n-1}^{(i)} \delta_{\alpha_{u, n \mid L\left(n^{\prime}-2\right)+l_{l}}^{(i)}}\left(\alpha_{u, n \mid L\left(n^{\prime}-2\right)+l_{l}}\right)
\end{aligned}
$$

where we detach the prior spatial PDF $f_{n \mid L\left(n^{\prime}-2\right)+l l}\left(\boldsymbol{\alpha}_{n}\right)$ into $U$ marginal distribution density functions, which belong to each channel. Then, we calculate sub-likelihood of each sub-particle $\alpha_{n \mid L\left(n^{\prime}-2\right)+l l}^{(i)}$ by the function eq. (12) and sort them in descending order. Finally, by integrating the associated sub-particle in each channel, we reconstruct the particles and recalculate its likelihood 


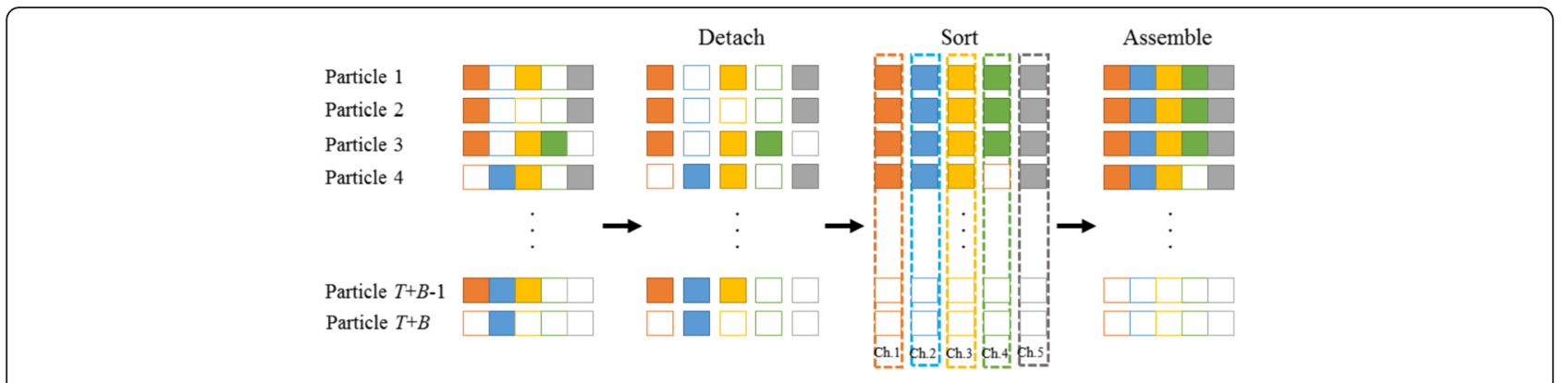

Fig. 4 Process of reducing complexity: different colors identify the sub-particles belonging to different channels; the solid squares signify the optimal sub-particle whose sub-likelihood ratio value is larger than others, while the hollow ones represent the other sub-optimal or worse sub-particles

function using eq. (11). With the above scheme, we just consider the $K$ kinds of particles in one channel avoiding the exponential growth of particles and a majority of particles of $f_{n \mid n}\left(\boldsymbol{\alpha}_{n}\right)$ will focus on optimal value after resampling.

Although we minimize the particle numbers by ignoring sub-optimal particles, this scheme cuts across the principle of diversity of particles in particle filters. Nevertheless, this is not the case. In the case of independent channels, variety of particles in CSI vector depends on each channel and such polarization does not cause the decline in sub-particle variety. Hence, it is entirely appropriate to match the PF principle.

\section{Simulation}

In this section, we will present several cooperative experiments in the realistic TVFF channels. Without loss of generality, we assume the channel states follow the commonly time-varying Rayleigh fading. Therefore, the $\mathrm{PDF}$ of channel fading gain is given by

$$
f_{R}(\alpha)=\frac{\alpha}{\sigma_{R}^{2}} \exp \left(-\frac{\alpha^{2}}{2 \sigma_{R}^{2}}\right)
$$

where $\sigma_{R}^{2}$ is the variance of Rayleigh fading.

Here, we configure the variance $\sigma_{R}^{2}=0.1$, the number of discrete channel state $K=5$. It is apparent that the CSS algorithm is operated recursively under the Bayesian criterion and thus defines the total detection probability $P_{D}$, which is adopted as a metric in [21], which considers the correct detection in both hypotheses $H_{1}$ and $H_{0}$ :

$$
P_{D}=1-p\left(H_{1}\right) P_{m}-p\left(H_{0}\right) P_{f}
$$

Besides, the mean square error (MSE) is defined as the other criterion to measure the performance of CSI. The equation is given by

$$
\operatorname{MSE}=\frac{1}{N} \sum_{n=1}^{N}\left[\sum_{i=1}^{I}\left(\hat{\alpha}_{i, n}-\alpha_{i, n}\right)^{2} / \sum_{i=1}^{I} \alpha_{i, n}^{2}\right]
$$

\subsection{Different SU_R numbers}

In the first experiment, performance of $P_{D}$ and MSE has been fully investigated from different numbers of SU_Rs. In the simulation, the length of pilot signal $M$ is configured to 100 . The transitional probability $\left(p_{b}, p_{s}\right)$ is set to $(0.4,0.7)$. The static length of $L$ is configured to 20 . We set the number of survival particle $T$ and birth particle $B$ to 50 and 50 .

Figure 5 shows the total detection probability curves of the CSS algorithm with different numbers of SU_Rs $U=3$, 5 , and 7. It is noted that the $P_{D}$ will be increased with increasing SNR, which is finally up to 1 . It is comprehensible that the more SU_Rs cooperate, the better performance of $P_{D}$ is shown. That is because with more SU_Rs, the FC is able to obtain more observations about the P_BS; as a result, the accuracy of sensing will be enhanced.

MSE curves have been shown in Fig. 6 in detail. Similar to detection curves in Fig. 5, the performance of MSE also becomes better with more SU_Rs and they will be convergent to the same value with increasing of SNR.

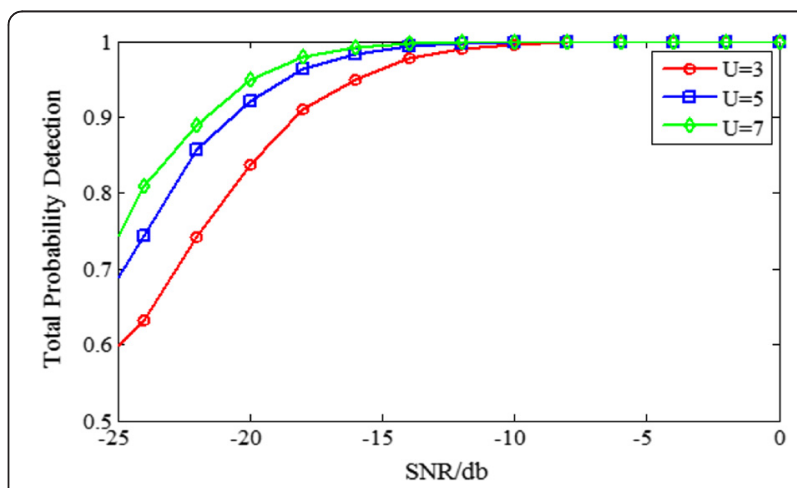

Fig. 5 Sensing performance of the CSS algorithm under different numbers of SU_R 


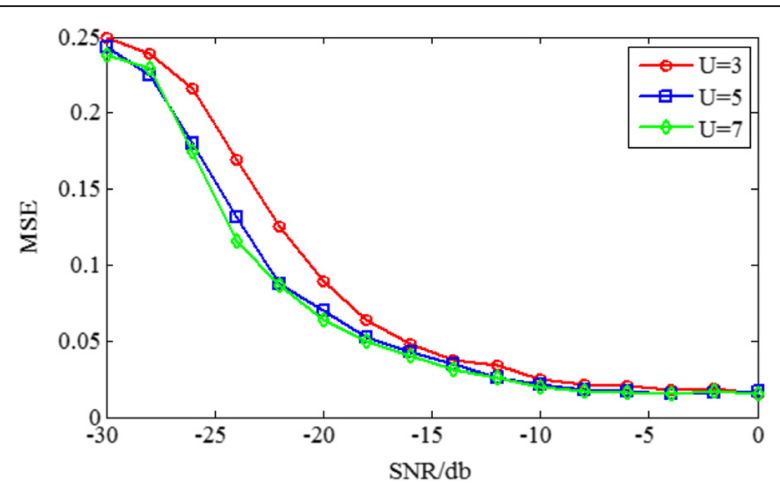

Fig. 6 MSE of the CSS algorithm under different numbers of SU_R

Note that, however, a compromise should be taken into account, since a more SU_R also results in a more complexity of CSI. For example, in Fig. 6 the increased performance from $U=5$ to $U=7$ is far less than that from $U=3$ to $U=5$.

\subsection{Comparative analysis in sensing performance}

For a comparative analysis, a classical optimal soft combination scheme has been entirely investigated in [10]. In this simulation, the number of SU_Rs is set to 5, the static length $L$ is 20, and length of pilot signal is 100 . The threshold in the classical scheme can be obtained according to the observation model in this paper. With $P_{f}=0.1$, its detection probability $P_{d}=\operatorname{Pr}\left\{L R\left(\mathbf{y}_{n}\right)>h \mid H_{1}\right\}$ curves have been shown in Fig. 6. Since the CSS algorithm belongs essentially to the Bayesian rule, in order to maximum the total detection probability $P_{D}$, the false alarm probability has been hidden in the $P_{D}$, where $P_{f}$ will be fixed hardly. Nevertheless, for comparing sensing performance of two schemes, a cooperative joint scheme can still be evaluated under the NP criterion. The blue solid curve belonging to the new scheme gains an advantage over the soft combination scheme with a fixed $P_{f}=0.1$.

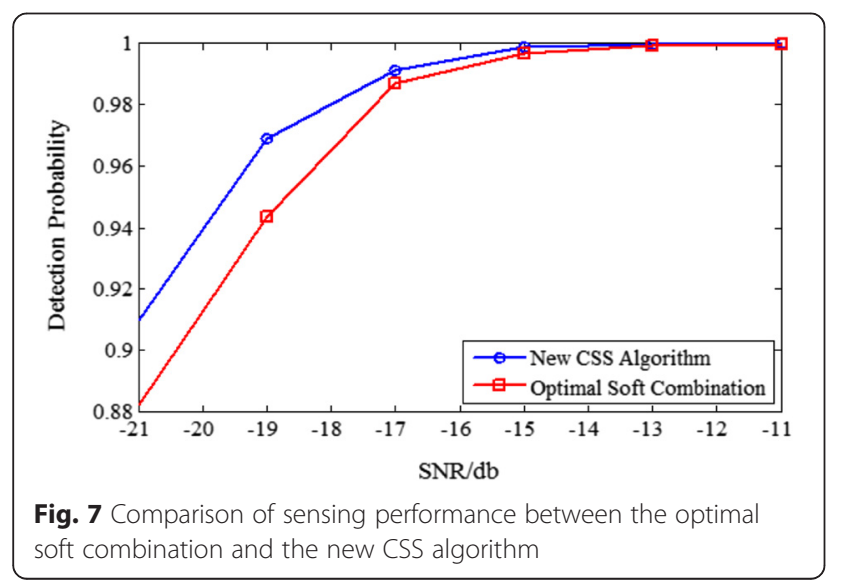

The new CSS scheme is superior to the classical one, obviously, since precise estimation of CSI can ensure the accuracy of likelihood function ratio between two hypotheses $H_{1}$ and $H_{0}$ (Fig. 7). It is crucial that such estimated CSI is surely responsible for the communication quality of cognitive link as well.

\section{Conclusions}

This work presents a new framework to reach dynamic spectrum sharing in TVFF channels. The central question addressed in the paper is how to effectively detect the occupancy of PU's band and estimate the CSI jointly. Based on a BF scheme, we design a joint CSS algorithm which achieves harmony and complementarity of detecting and estimating. Experimental simulation has validated our new scheme clearly. This new scheme also provides a promising solution to sense other dynamic parameters like the location of primary users in CRN. By casting these parameters into the DSM, they can be estimated recursively together by the BF algorithm. Thus, the new CSS is accessible to a more realistic CRN.

\section{Competing interests}

The authors declare that they have no competing interests.

\section{Acknowledgements}

This work was supported by NSFC (61379016) and SRFDP (20130005110016).

Received: 30 September 2015 Accepted: 24 February 2016

Published online: 10 March 2016

\section{References}

1. Q Liang, Automatic target recognition using waveform diversity in radar sensor networks. Pattern Recogn Lett 29(3), 377-381 (2008)

2. Q Liang, Waveform design and diversity in radar sensor networks: theoretical analysis and application to automatic target recognition. In sensor and ad hoc communications and networks, 2006. SECON'06. 2006 3rd annual IEEE communications society on Vol. 2 (IEEE, Reston, VA, 2006), pp. 684-689

3. Q Liang, X Cheng, SC Huang, D Chen, Opportunistic sensing in wireless sensor networks: theory and application. IEEE Trans Comput 63(8), 20022010 (2014)

4. S Singh, Q Liang, D Chen, L Sheng, Sense through wall human detection using UWB radar. EURASIP J Wirel Commun Netw 2011(1), 1-11 (2011)

5. S. Haykin, Cognitive radio: brain-empowered wireless communications. Selected Areas in Communications, IEEE J 23(2), 201-220 (2005)

6. Federal Communications Commission, Spectrum policy task force report, FCC 02-155 (2002)

7. E Axell, G Leus, EG Larsson, HV Poor, Spectrum sensing for cognitive radio: stateof-the-art and recent advances. IEEE Signal Processing Mag 29(3), 101-116 (2012)

8. C.R. Stevenson, C. Cordeiro, E. Sofer, G. Chouinard, Functional requirements for the 802.22 WRAN standard: IEEE 802.22-05/0007r46 (IEEE, Piscataway, 2005)

9. IEEE, Draft supplement to standard for telecommunications and information exchange between systems_-LAN/MAN specific requirements_-Part 11 (2003) wireless medium access control (MAC) and physical layer (PHY) specifications: specification for radio resource measurement: IEEE $802.11 \mathrm{k}$ | D0.7 (IEEE, Piscataway, 2003)

10. J Ma, G Zhao, Y Li, Soft combination and detection for cooperative spectrum sensing in cognitive radio networks. IEEE Trans Wirel Commun 7(11), 45024507 (2008)

11. SM Mishra, A Sahai, RW Brodersen, Cooperative sensing among cognitive radios. In Communications, 2006. ICC'06. IEEE International Conference on Vol. 4. (IEEE, Istanbul, 2006), pp. 1658-1663

12. Z Quan, S Cui, AH Sayed, Optimal linear cooperation for spectrum sensing in cognitive radio networks. IEEE J Selected Top Signal Processing 2(1), 28-40 (2008) 
13. FF Digham, MS Alouini, MK Simon, On the energy detection of unknown signals over fading channels. In Communications, 2003. ICC'O3. IEEE international conference on Vol. 5. (IEEE, 2003), pp. 3575-3579

14. M Bkassiny, Y Li, SK Jayaweera, A survey on machine-learning techniques in cognitive radios. IEEE Commun Surv Tutor 15(3), 1136-1159 (2013)

15. W Lewandowski, J Azoubib, WJ Klepczynski, GPS: primary tool for time transfer. Proc IEEE 87(1), 163-172 (1999)

16. P Sadeghi, RA Kennedy, PB Rapajic, R Shams, Finite-state Markov modeling of fading channels-a survey of principles and applications. IEEE Signal Processing Mag 25(5), 57-80 (2008)

17. C Zhao, M Sun, B Li, L Zhao, X Peng, Blind spectrum sensing for cognitive radio over time-variant multipath flat-fading channels. EURASIP J Wirel Commun Netw 2014(1), 1-13 (2014)

18. RP Mahler, Statistical multisource-multitarget information fusion. (Artech House, Inc., Norwood, MA, USA, 2007)

19. B Ristic, BT Vo, BN Vo, A Farina, A tutorial on Bernoulli filters: theory, implementation and applications. IEEE Trans Signal Processing 61(13), 3406-3430 (2013)

20. B Ristic, S Arulampalam, Bernoulli particle filter with observer control for bearings-only tracking in clutter. IEEE Trans Aerosp Electron Syst 48(3), 2405-2415 (2012)

21. B Li, S Li, A Nallanathan, Y Nan, C Zhao, Z Zhou, Deep sensing for nextgeneration dynamic spectrum sharing: more than detecting the occupancy state of primary spectrum. IEEE Trans Commun 63(7), 2442-2457 (2015)

\section{Submit your manuscript to a SpringerOpen ${ }^{\odot}$ journal and benefit from:}

- Convenient online submission

- Rigorous peer review

- Immediate publication on acceptance

Open access: articles freely available online

- High visibility within the field

- Retaining the copyright to your article

Submit your next manuscript at $\boldsymbol{s p r i n g e r o p e n . c o m ~}$ 